\title{
«Je m'inscris au cours de formation continue. Je suis végétarien.»
}

\section{Hermann Amstad ${ }^{a}$, Walter Reinhart ${ }^{b}$}

a Dr, secrétaire général de l'ASSM, Bâle

b Prof. Dr, médecin-chef médecine interne, Hôpital cantonal de Coire, vice-président de l'ASSM

* La «Commission consultative» est composée des personnalités suivantes: Prof. Walter Reinhart, Coire (président); Dr Gilbert Abetel, Orbe; Prof. Anne-Françoise Allaz, Genève; Dr Hermann Amstad, Bâle; Prof. Jerôme Biollaz, Lausanne; Dr Dieter Grauer, Zurich;

Prof. Reto Krapf, Bruderholz; Prof. Urban Laffer, Bienne; Prof. Thomas Lüscher, Zurich; Dr Christian Marti, Winterthour; Dr Alain Michaud, Nyon; Dr Jürg Müller, Bâle; Prof. Reto Obrist, Sion; Valérie Rothhardt, avocate, Berne; Dr Urs Strebel, Männedorf.

\section{Correspondance:}

Dr Hermann Amstad

Académie des Sciences Médicales

Suisses

Petersplatz 13

CH-4051 Bâle

h.amstad@samw.ch
En 2002, l'Académie Suisse des Sciences Médicales (ASSM) a publié pour la première fois des recommandations pour la «Collaboration corps médical - industrie». Remaniées en 2005, les directives «Collaboration corps médical - industrie» font désormais partie du code déontologique de la FMH, rendant ainsi leur observation obligatoire pour tous les médecins suisses.

Afin de faciliter et d'encourager la mise en pratique de ces directives, le Sénat de l'ASSM a créé en mai 2007 un «poste de consultation» et une «commission consultative» dirigée par le Prof. Walter Reinhart de Coire, vice-président de l'ASSM. Le poste de consultation répond aux questions et reçoit les annonces relatives à l'application des directives. Le rôle de la commission consultative* est de soutenir le poste de consultation en apportant des clarifications et des prises de position fondamentales, en complétant les directives et en délivrant des avis publics. Elle observe la jurisprudence de la Confédération et, le cas échéant, prend position. Par ailleurs, elle est tenue de rendre compte de ses activités chaque année.

De juillet 2007 à juin 2008, la commission consultative a siégé trois fois (le 28 août 2007, le 9 janvier 2008, le 30 avril 2008,) en concentrant son travail sur les points suivants:

- le traitement de diverses demandes;

- l'information des organisateurs de cours de formation continue sur la non-observation des directives;

- les règles de conduites de la commission.

Au-delà de ces réunions, la commission consultative a rencontré une délégation d'une grande société de discipline médicale pour un échange d'expériences.

\section{Traitement des demandes}

Au courant de l'année, diverses demandes concernant des thèmes variés nous sont parvenues, soit par courrier, soit par e-mail, tant de la part de médecins individuels que de sociétés de discipline médicale et d'entreprises. Certaines d'entre elles que la commission consultative estime fondamentales - sont résumées ci-après.

\section{Exclusion de concurrents du sponsoring}

Une société de discipline médicale organise chaque année trois cours de formation continue sponsorisés par 5 à 6 entreprises pharmaceutiques; les participants paient une contribution adaptée. Lors du démarrage du premier cours en 1999, une entreprise a offert de soutenir le cours avec 30000 francs par an; en contrepartie, la société de discipline médicale a promis verbalement à l'entreprise de ne prendre en considération aucun autre sponsor ayant un portfolio comparable. Dans l'intervalle, cette réglementation a été critiquée par un concurrent et la responsable du cours de formation continue a, elle-même, eu l'impression que les directives «Collaboration corps médical - industrie» ne permettaient plus cette forme de (mono)sponsoring.

Après un examen approfondi de la situation, la commission conclut que le règlement tel qu'il avait été décidé en 1999 était tout à fait fondé, mais qu'il est devenu obsolète avec l'entrée en vigueur des directives «Collaboration corps médical - industrie» en 2006. Selon la commission, le fait d'exclure les autres concurrents équivaut au monosponsoring; même si les directives ne le stipulent pas explicitement, ces pratiques ne correspondent pas à leur «philosophie». De plus, les directives précisent que tous les accords avec les sponsors doivent être faits par écrit (sous forme contractuelle). Cette disposition offre alors une bonne occasion de prendre contact avec son sponsor pour envisager de nouveaux accords. La commission est persuadée qu'il est possible de trouver un consensus; elle considère les exigences d'exclusivité des entreprises pharmaceutiques comme des menaces dépourvues de fondement.

\section{Soutien par des fonds provenant de tiers}

Un représentant d'une entreprise de produits médicaux demande si les directives comprennent des consignes concernant le soutien par des fonds provenant de tiers resp. le «sponsoring de la recherche soutenue par des fonds provenant de tiers». Dans le premier cas, l'entreprise finance un collaborateur à temps partiel dans un service hospitalier; dans le deuxième cas, l'entreprise 
met à la disposition d'un hôpital des moyens financiers pour la recherche, sans qu'il y ait de projet concret.

Dans les deux cas exposés, la commission a conclu qu'elle ne voyait pas d'objection fondamentale. Ces activités de sponsor ne posent pas de problème tant que le chapitre III des directives («Acceptation de prestations en espèces ou en nature») est respecté. Celui-ci précise les points suivants:

- une distinction stricte doit être faite entre l'acceptation de prestations en espèces ou en nature et les achats de l'institution;

- tous les accords relatifs à l'acceptation de telles prestations doivent être consignés par écrit (sous forme contractuelle);

- lorsqu'il s'agit de sommes importantes, ce contrat requiert une signature de la direction de l'hôpital (principe de surveillance mutuelle);

- le contrat contient les affectations des fonds versés ainsi que l'assurance qu'aucun accord annexe (oral ou tacite) n'a été convenu.

\section{Organisation de cours de formation continue par des entreprises pharmaceutiques}

La responsable de la formation continue d'une société de discipline médicale sollicite l'avis de la commission sur le fait que des entreprises pharmaceutiques projettent des cours de formation continue sur un thème médical correspondant à leur gamme de produits et introduisent en même temps (pour garantir leur indépendance professionnelle) un Advisory Board avec des spécialistes et un représentant de la médecine de premier recours. Elle pense que le monosponsoring et le choix des conférenciers et des sujets ne sont pas conformes aux directives de l'ASSM «Collaboration corps médical - industrie»; en même temps, elle a l'impression d'être la seule personne à s'en formaliser et aimerait savoir si l'ASSM envisage des mesures destinées à freiner cette tendance.

La commission partage son avis en ce qui concerne la non-observation des directives dans les pratiques relatées. A plusieurs reprises, l'ASSM a rendu les sociétés de discipline médicale attentives (par courrier et dans le Bulletin des médecins suisses) au fait que de telles manifestations ne sont pas reconnues comme formation continue et ne donnent donc pas droit à des crédits. Les sociétés de discipline médicale ont donc la responsabilité de veiller à ce que leurs membres puissent acquérir le nombre de crédits nécessaire dans des cours de formation continue reconnus.

Au-delà de la question de la reconnaissance des cours de formation se pose la question de la qualité: la commission est convaincue qu'il manque aux manifestations organisées par l'industrie pharmaceutique la pondération nécessaire et qu'elles ne satisfont pas aux exigences de qualité attendues par des médecins à l'esprit critique.

\section{Monosponsoring}

Selon le chapitre 2, les manifestations soutenues par un seul sponsor ne devraient en principe pas être reconnues comme formation continue; ce n'est que dans certains cas justifiés que le sponsoring par une seule entreprise peut être possible. Un collaborateur d'une société spécialisée dans l'organisation de congrès demande ce que l'on entend par «certains cas justifiés». La commission consultative prévoit de publier, encore au courant de cette année, une aide à l'interprétation.

\section{Participation aux frais}

L'organisateur d'un cours de formation continue, établi depuis des années, gratuit et très prisé, est confronté au problème de la contribution financière des participants comme prévu dans les directives. Il pense que le travail supplémentaire causé par la perception de frais de participation serait considérable, car jusqu'à ce jour les inscriptions n'étaient pas comptabilisées et il n'y avait aucun contrôle au niveau des entrées.

La commission consultative accepte comme compromis provisoire de ne délivrer des confirmations de la participation que contre l'acquittement d'un droit d'inscription. Toutefois, en principe, cette pratique est contraire aux directives (chiffre 6: «There is no free lunch») et exige une adaptation dans ce sens. La commission estime que le travail causé par l'expédition de bulletins de versement ou la saisie des numéros de compte chèque postaux - sans contrôle lors de la manifestation elle-même - est acceptable. Par ailleurs, les médecins en formation et les retraités peuvent être exemptés des frais d'inscription.

\section{Information des organisateurs de cours de formation continue sur la non-observation des directives}

Au courant de l'année, le poste de consultation a reçu de nombreuses annonces de cas de nonobservation des directives. Divers cours de formation continue étaient manifestement monosponsorisés; de même, les cours étaient souvent proposés par les entreprises pharmaceutiques ellesmêmes. Quelquefois aussi, des cours de plus d'une demi-journée étaient donnés gratuitement, ce qui est contraire aux directives. Plutôt amusant, le cas d'un cours de formation continue sur le traitement des patients souffrant de maladies cardiovasculaires suivi d'un cours de cuisine! Une rubrique «végétarien: oui / non» était prévue sur le talon d'inscription, d'où le titre de ce rapport.

Dans toutes ces situations, le poste de consultation (en accord avec le président) a contacté les organisateurs et les sociétés de discipline médi- 
cale pour les rendre attentifs aux directives et les prier de changer leur pratique.

Dans un autre cas, une entreprise pharmaceutique a seulement indiqué son nom (et ses finances) pour une série de manifestations, toutes sous la responsabilité de la clinique organisatrice et dont la qualité était incontestée. La commission a décidé de vérifier une nouvelle fois ses recommandations concernant cette forme de monosponsoring lors de sa prochaine séance.

Les membres de la commission ont discuté les possibilités d'améliorer l'observation des directives et ont proposé que les organisateurs de cours de formation continue stipulent sur le programme - comme une «autodéclaration» que les directives «Collaboration corps médical industrie » sont respectées.

\section{Règles de procédures \\ de la commission consultative}

La commission a jugé utile d'élaborer certaines règles de procédures pour le traitement des demandes. A cet égard, les points suivants ont été considérés comme particulièrement importants:

- les demandes resp. réclamations devraient être saisies de façon centralisée et systématique (auprès du poste de consultation) et traitées ensuite de façon structurée;

- le poste de consultation doit réagir directement lorsqu'il s'agit de «cas typiques» (p.ex. les cas fréquents de monosponsoring des cours de formation); par contre les cas plus complexes doivent être transmis à la commission consultative;

- il conviendra de discuter si les décisions resp. les recommandations de la commission consultative doivent être approuvées à l'unanimité ou - dans le cas contraire - si les positions minoritaires doivent être signalées dans l'optique d'une information transparente.

La crédibilité de la commission consultative est à l'image de la réputation de ses membres. C'est pourquoi une attitude transparente face à leurs éventuelles relations d'intérêts est souhaitable. La commission a donc décidé que tous ses membres signalent leurs relations d'intérêts par rapport à l'industrie (actionnariat, conseil d'administration, mandat de conseiller); ces informations ne sont toutefois pas publiques, et ne sont divulguées que sur demande.

\section{Problèmes rencontrés par une importante société de discipline médicale dans la mise en œuvre des directives «Collabo- ration corps médical - industrie»}

Lors de sa troisième séance, la commission a reçu une délégation d'une importante société de dis- cipline médicale pour une discussion principalement axée sur les points suivants:

- la réglementation pour la formation continue (RFC) de la FMH prévoit certaines consignes relatives à la structure et à la reconnaissance des cours de formation continue; les programmes de formation continue des sociétés de discipline médicale précisent ces consignes. A cet égard, chaque société de discipline médicale se demande ce qu'elle doit imposer resp. contrôler dans les cours de formation continue prévus par la législation;

- ou plus fondamentalement: le «devoir de formation continue», tel qu'il est défini par l'article 40 de la loi sur les professions médicales, se limite-t-il à justifier le nombre d'heures passées en cours de formation?

- actuellement il reste quelques zones d'ombres dans le règlement concernant la reconnaissance des cours de formation continue. Les différentes sociétés de discipline médicale ont des pratiques variables, ce qui provoque un certain agacement chez l'un ou l'autre de leurs membres;

- par ailleurs, il reste à déterminer si les «crédits» pour la participation aux cours doivent être octroyés seulement sur la base du critère quantitatif («une heure de formation continue») ou si des éléments qualitatifs doivent également être pris en compte. La commission consultative ne peut ni ne doit s'exprimer quant au nombre de crédits accordés par cours de formation continue;

- c'est la FMH qui doit régler la question de la validation de cours de formation continue. Comment éviter que des médecins se fassent «acheter»?

- il n'existe aucun système exempt d'abus. Un institut de formation postgraduée ne peut pas garantir à l'avance qu'il est indépendant;

- les comités de direction des sociétés de discipline médicale devraient procéder à une enquête auprès de leurs membres pour encourager l'observation conséquente des directives; - beaucoup de médecins seraient prêts à payer pour des cours de formation continue de qualité.

\section{Perspectives}

Au courant de cette première année d'exercice, la commission consultative avait encore fort à faire pour définir sa propre identité. A l'avenir, elle aimerait s'orienter davantage vers l'extérieur pour mieux se faire connaître. La commission consultative aimerait encourager les médecins à régler euxmêmes leurs relations quelquefois délicates avec l'industrie (de la santé) au lieu d'y être contraints par des tiers sous quelque forme que ce soit. 